\title{
Specific detection of soluble EphA2 fragments in blood as a new biomarker for pancreatic cancer
}

\author{
Naohiko Koshikawa ${ }^{\star, 1,2}$, Tomoko Minegishi ${ }^{1,2}$, Hirofumi Kiyokawa ${ }^{1,3}$ and Motoharu Seiki ${ }^{2,4}$
}

Because membrane type 1-matrix metalloproteinase 1 (MT1-MMP) and erythropoietin-producing hepatocellular receptor 2 (EphA2) expression are upregulated by the Ras/mitogen-activated protein kinase pathway, they are frequently coexpressed in malignant tumors. MT1-MMP cleaves the N-terminal ligand-binding domain of EphA2 and inactivates its ligand-dependent tumor-suppressing activity. Therefore, specific detection of the cleaved N-terminal EphA2 fragment in blood might be an effective biomarker to diagnose malignant tumors. To evaluate this possibility, we developed three monoclonal antibodies against the soluble EphA2 fragment. One of them recognized this fragment specifically, with negligible cross-reactivity to the intact form. We used the cleaved form-specific antibody to develop a quantitative enzyme-linked immunosorbent assay and confirmed the linear reactivity to the recombinant fragment. We applied this assay on commercially available serum specimens obtained from patients with several types of cancer including gastric, pancreatic, esophageal, gastroesophageal, and head-and-neck cancers, and healthy donors. Soluble EphA2 fragment levels in cancer-patient sera were higher than those in healthy donors $(n=50)$. In particular, levels of eight out of nine (89\%) pancreatic cancer patients and ten out of seventeen (59\%) gastric cancer patients significantly exceeded cutoff values obtained from the healthy donors, whereas those of esophageal and head-and-neck cancer-patient sera were low. The preliminary receiver operating characteristic curve analysis for pancreatic cancer demonstrated that the sensitivity and specificity were $89.0 \%$ and $90.0 \%$, respectively, whereas those of the conventional digestive tumor marker CA19-9 were $88.9 \%$ and $72.0 \%$, respectively. These results indicated that specific detection of soluble EphA2 fragment levels in serum could be potentially useful as a biomarker to diagnose pancreatic cancer.

Cell Death and Disease (2017) 8, e3134; doi:10.1038/cddis.2017.545; published online 26 October 2017

Erythropoietin-producing hepatocellular receptor 2 (EphA2), a member of the mammalian Eph receptor kinase family, is expressed in epithelial cells and acts to suppress unscheduled growth signals for stabilization of the epithelial cell phenotype. In addition, EphA2 is expressed in vascular endothelial cells and has a role in tumor vessel formation through the stimulation of cell migration. ${ }^{1,2}$ Furthermore, it is also overexpressed frequently in different types of cancers such as breast, liver, pancreatic, prostate, esophageal, glioma, melanoma, and ovarian adenocarcinoma. ${ }^{3}$ Because overexpression of EphA2 is associated with disease progression and metastasis of cancer cells, and it is thought to be a possible target for cancer therapy, ${ }^{3,4}$ small-molecule inhibitors, siRNAs, and neutralizing antibodies against EphA2 are currently under evaluation while leaving the possibility of EphA2 as a tumor suppressor.

Recently, it became clear that EphA2 regulates ErbB receptor-mediated signals differently depending on availability of its ligands, ephrins. In the presence of ephrins, EphA2 suppresses ErbB receptor signals, whereas ligandindependent EphA2 signals cooperate with ErbB receptormediated signals to promote tumor growth and invasion. ${ }^{5}$ Therefore, the availability of ligands appears to determine which function of EphA2 predominates during tumor progression.

EphA2 ligands (for example, ephrin-A1) are expressed abundantly in different tumor types such as breast carcinoma and melanoma, and normal tissues. ${ }^{6}$ Soluble ephrin-A1 is released by cleavage of matrix metalloproteinases (MMPs) and is detected in the sera of patients with hepatocellular carcinoma. $^{7,8}$ Therefore, critical questions are how and when ligand-independent EphA2 signaling is activated in tumors.

We have demonstrated recently a possible mechanism involving proteolytic processing to convert EphA2 into a ligand-insensitive form. ${ }^{9-11}$ Membrane type 1-MMP (MT1MMP) is a membrane MMP that is frequently overexpressed in malignant tumors. MT1-MMP regulates tumor progression via processing of bioactive proteins and extracellular matrices on the cell periphery, as well as through activation of hypoxiainducible transcription factors in a cytoplasmic tail-mediated manner. ${ }^{12-14}$ The expression of both EphA2 and MT1-MMP is upregulated by the Ras/mitogen-activated protein kinase (MAPK) pathway and is frequently observed on cancer cell membranes. ${ }^{15,16}$ EphA2 is an MT1-MMP substrate. Its cleavage by MT1-MMP eliminates the ligand-binding domain and converts it into a ligand-unavailable form by inactivating its tumor-suppressor activity. ${ }^{9,10}$

\footnotetext{
${ }^{1}$ Division of Cancer Cell Research, Kanagawa Cancer Center Research Institute, Yokohama, Japan; ${ }^{2}$ Division of Cancer Cell Research, Institute of Medical Science, University of Tokyo, Tokyo, Japan; ${ }^{3}$ Division of Gastroenterology and Hepatology, St. Marianna University School of Medicine, Kawasaki, Japan and ${ }^{4}$ Faculty of Medicine, Institute of Medical, Pharmaceutical, and Health Sciences, Kanazawa University, Kanazawa, Japan

*Corresponding author: N Koshikawa, Division of Cancer Cell Research, Kanagawa Cancer Center Research Institute, 2-3-2 Nakao Asahi-ku, Yokohama 241-8515, Japan. Tel: +81 45520 2222; Fax: +81 45520 2216; E-mail: nkoshi-tky@umin.ac.jp

Received 30.5.17; revised 08.9.17; accepted 13.9.17; Edited by M Piacentini
} 
Immunohistochemical analysis of several cancer tissues revealed that EphA2 coexpressed with MT1-MMP in several carcinoma tissues mostly lacked the $\mathrm{N}$-terminal ligand-binding domain, and activated ligand-independent EphA2 signaling (phosphorylation of Ser $\left.{ }^{897}\right)^{5,9}$ Forced expression of an uncleavable EphA2 mutant in carcinoma cells altered cell morphology to epithelial-like forms, and suppressed tumor growth and lung metastasis in mice. Therefore, processing of EphA2 by MT1-MMP in cancer cells represents an important cancer progression-related event. If the cleaved $\mathrm{N}$-terminal domain of EphA2 is released from cells and circulates stably in patient blood, it could be a useful biomarker for early detection in cancer patients using a conventional blood test. During preparation of this manuscript, another group reported that serum EphA2 could be a potential biomarker to diagnose pancreatic cancer, although that report made no mention of the form of EphA2. ${ }^{17}$

In the current study, we developed monoclonal antibodies (mAbs) against the soluble EphA2 fragment released from cancer cells by MT1-MMP cleavage. Several clones were obtained, with one showing reactivity to the soluble EphA2 fragment and negligible cross-reactivity to the intact form. We used this fragment-specific antibody to develop a sandwich enzyme-linked immunosorbent assay (ELISA) and applied it to measure levels of the soluble EphA2 fragment in sera from cancer patients.

\section{Results}

Purification of recombinant antigens. The extracellular portion of EphA2 following the signal peptide (1-27 amino acids (aa)) is composed of the ligand-binding domain (28-201 aa), the cysteine-rich domain (202-328 aa), and the stem region (329-537 aa) from the $\mathrm{N}$ terminus (Figure 1a). We and others mapped the cleavage sites of EphA2 by MT1-MMP in the stem region as indicated by arrows in Figure 1a. ${ }^{9,18}$ For immunization to raise mAbs, we prepared recombinant antigens spanning 28-328 aa (antigen $\# 1,75 \mathrm{kDa}$ ) and 28-537 aa (antigen \#5, $75 \mathrm{kDa}$ ) by expressing, respectively, the 1-328 and 1-537 regions in FreeStyle 293-F (Thermo Fisher Scientific, Waltham, MA, USA) and A431 cells as FLAG-tagged forms at the C terminus (Figure 1a). The antigens were purified and confirmed by western blotting using an anti-FLAG mAb. We prepared three additional FLAG-tagged antigens (\#2, 3, and $4 ; 40,50$, and $50 \mathrm{kDa}$, respectively) by expressing them in Escherichia coli as fusion proteins with an $\mathrm{N}$-terminal
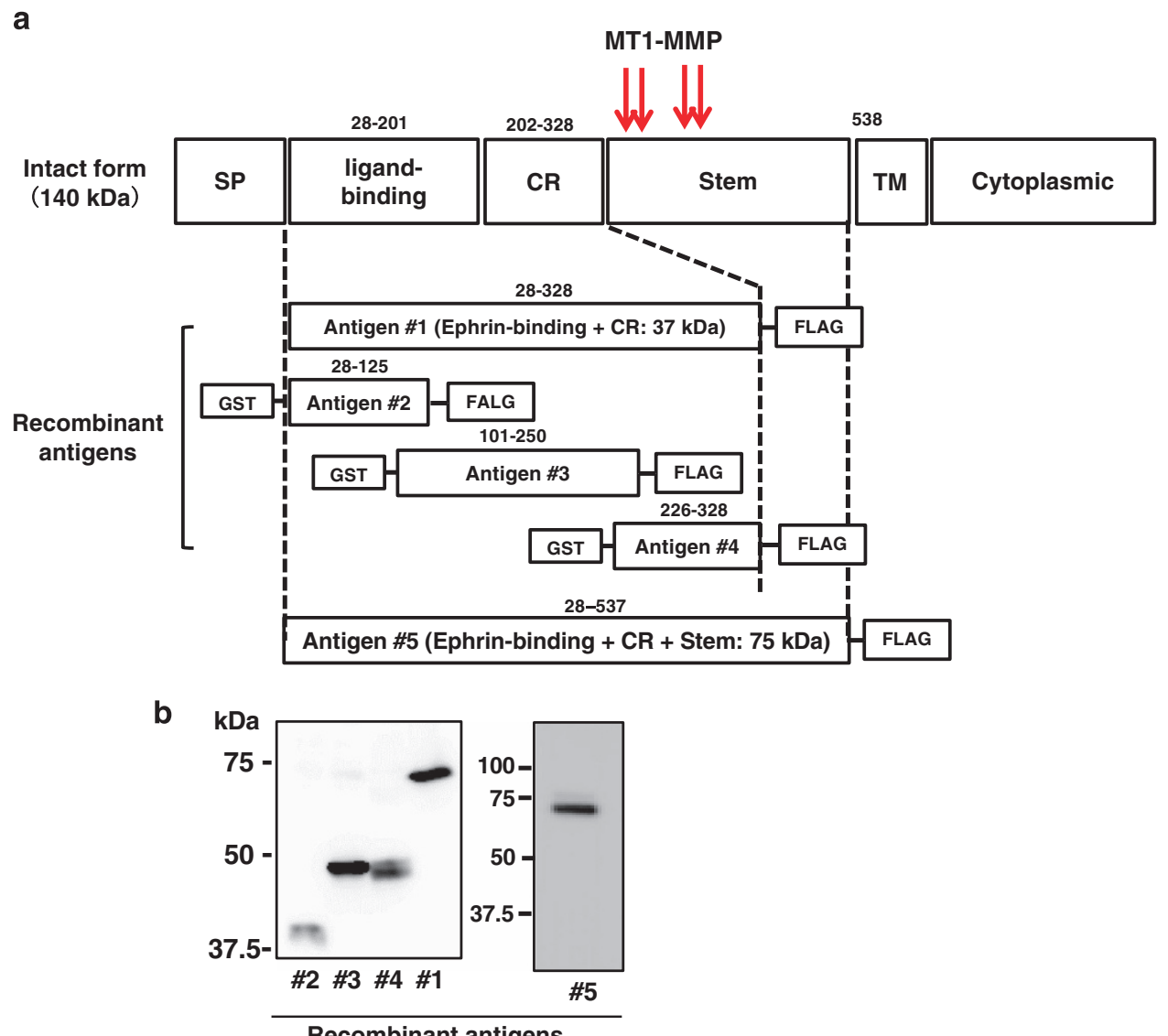

Figure 1 Preparation of recombinant EphA2 and its fragments. (a) Intact EphA2, signal peptide (SP), ligand-binding, cysteine-rich (CR), stem, transmembrane (TM), and cytoplasmic domains are denoted. Red arrows indicate the cleavage sites by MT1-MMP. Recombinant antigens \#1 and 5 were expressed with the C-terminal FLAG-tag in FreeStyle 293-F or A431 cells as secreted proteins in conditioned medium. Antigens \#2, 3, and 4 were produced in Escherichia coli. (b) All antigens were analyzed by western blot using an anti-FLAG mAb under reducing conditions after purification on an anti-FLAG mAb-conjugated agarose column. GST: glutathione S-transferase 
glutathione S-transferase tag. Each antigen was purified and detected as before (Figure 1b).

Reactivity of mAbs against the cleaved form of the EphA2 fragment. Mice were immunized with antigen \#1 $(5 \mu \mathrm{g} /$ mouse $)$ and 400 fused cells were obtained. The recovered mAbs were screened by ELISA using plates coated with antigen \#1 (0.5 $\mu \mathrm{g} /$ plate). Three different hybridoma clones (46A1, 62A1, and 76A1) that produced the $\lg _{1}$ class of $\mathrm{mAbs}$ were selected and analyzed further. To confirm reactivity of the mAbs, antigen \#1 was immunoprecipitated using them, or other available anti-EphA2 antibodies, and was subjected to $12.5 \%$ sodium dodecyl sulfate polyacrylamide gel electrophoresis (SDS-PAGE) under reducing conditions. This was followed by western blot analysis using an anti-FLAG mAb (Figure 2a). All three mAbs precipitated antigen \#1 similarly to the positive control antibodies (371805 and 96-1); however, negative control antibodies (mouse IgG and anti-FLAG $\mathrm{mAb}$ ) did not precipitate antigen \#1 (Figure 1a).

To map their epitopes, the four recombinant EphA2 fragments (Figure 1a, antigens \#1-4) were subjected to SDS-PAGE under reducing or non-reducing conditions and were analyzed with western blot analysis (Supplementary Figure S1). mAbs 62A1 and 76A1 detected antigen \#1 under reducing conditions (Figure $2 \mathrm{a}$ ), whereas $\mathrm{mAb} 46 \mathrm{~A} 1$ failed to detect this antigen in non-reducing conditions (Supplementary Figure S1). mAb 62A1 reacted with antigen \#3, but the two other mAbs did not react with antigens \#2, 3, or 4. This indicates that epitopes of the three new mAbs exist in an area of antigen \#1 that does not overlap with antigens \#2, 3, or 4.

To confirm reactivity of the mAbs to other forms of EphA2 carrying the reactive epitope, the entire extracellular domain (antigen \#5) was expressed in human epidermoid carcinoma A431 cells that also expressed endogenous EphA2. The cells were lysed and subjected to immunoprecipitation with the mAbs, and then western blotted under non-reducing conditions. Positive control antibodies (96-1, 371805, and C20) immunoprecipitated the intact form effectively, and the protein was detected by western blot analysis using the C20 mAb (Figure $2 b$ ). Interestingly, whereas $\mathrm{mAb} 46 \mathrm{~A} 1$ recognized the intact form, mAb 76-1 did not and 62A1 did so only weakly. It is of note that $\mathrm{mAb} 76 \mathrm{~A} 1$ also failed to recognize the whole EphA2 extracellular domain fragment (antigen \#5; Figure 2c). Therefore, mAb 76A1 appears to recognize the soluble EphA2 fragment in a fragment-specific manner rather than the intact and whole extracellular domain-containing forms. In contrast, mAbs $46 \mathrm{~A} 1$ and $62 \mathrm{~A} 1$ reacted with all EphA2 forms containing its epitope (intact EphA2, antigens \#1 and 5) and mAb 62A1 recognized antigens $\# 1$ and $\# 5$ (Figures $2 a$ and $c$ ). On the basis of these characterizations, mAb 76A1 is the most suitable to capture the soluble EphA2 fragment in blood and to develop a sandwich ELISA system as a diagnostic tool.

Analysis of the soluble EphA2 fragment in sera of cancer patients and healthy volunteers. A combination of two mAbs (76A1 and 46A1) was used for a sandwich ELISA; $76 \mathrm{~A} 1$ to capture antigen $\# 1$ and $46 \mathrm{~A} 1$ for detection. To examine the quantitative reactivity of this ELISA, we added increasing amounts of antigen \#1 $(0-250 \mathrm{pg} / \mathrm{ml})$ to the test

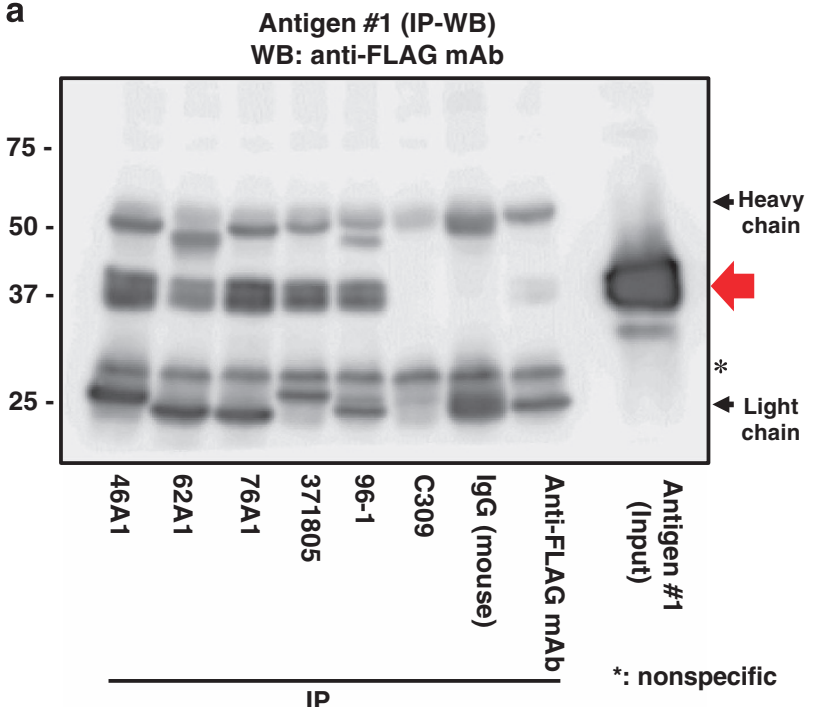

b

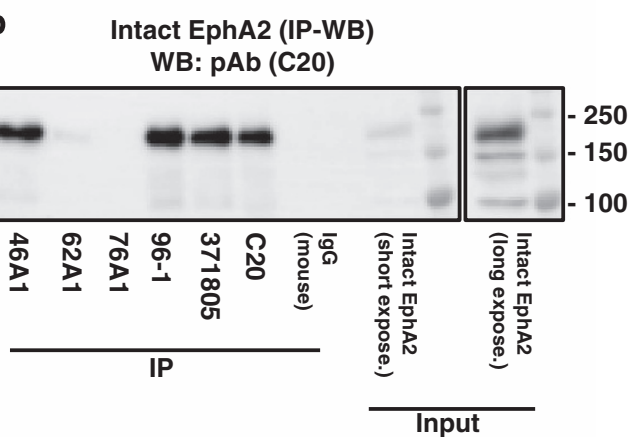

C Antigen \#5 (IP-WB) WB: pAb (C20)

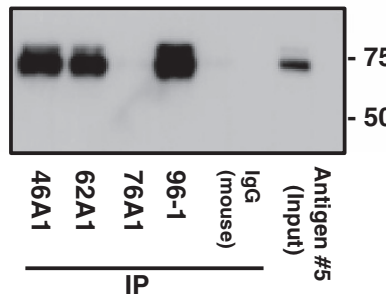

Figure 2 Immunoprecipitation of recombinant EphA2 domains and intact EphA2 protein with anti-EphA2 mAbs. EphA2 proteins expressed in cells were immunoprecipitated by the indicated mAbs. (a) The precipitated $37 \mathrm{kDa}$ band corresponding to antigen \#1 was detected by western blot using an anti-FLAG mAb (red arrow). This band was recognized by mAbs $46 \mathrm{~A} 1,62 \mathrm{~A} 1$, and $76 \mathrm{~A} 1$ immunized by antigen \#1. Antigen \#1 was precipitated weakly by the anti-FLAG mAb. Immunoglobulins (heavy and light chains) are indicated by black arrows. *Indicates a $30 \mathrm{kDa}$ nonspecific band. (b) Precipitated intact EphA2 in the A431 cell lysate was detected by western blot using an anti-EphA2 cytoplasmic domain pAb (C20). mAb 46A1 and positive control antibodies (96-1, 371805, and C20) reacted with intact EphA2. mAb 62A1 recognized intact EphA2 very faintly. In contrast, mAb 76A1 did not show any positive signal. The right panel shows a long exposure to intact EphA2 detected by pAb C20. (c) Precipitated antigen \#5 (extracellular domain of EphA2) was detected by western blot using an anti-EphA2 cytoplasmic domain polyclonal antibody (pAb) (C20). mAb 46A1, 62A1 and positive control antibody (96-1) reacted with antigen \#5, but mAb 76A1 did not. IP, immunoprecipitation; WB, western blot 
a

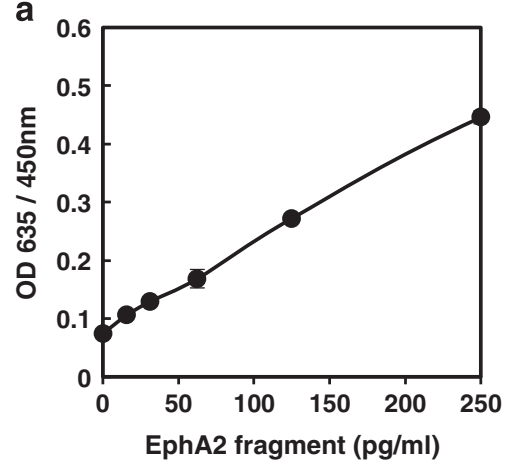

C

\begin{tabular}{|c|c|}
\hline Disease & positive ratio (\%) \\
\hline \hline Gastric carcinoma & $59(n=17)$ \\
\hline Pancreatic carcinoma & $89(n=9)$ \\
\hline Esophagus carcinoma & $12.5(n=8)$ \\
\hline Gastroesophagus carcinoma & $100(n=1)$ \\
\hline head and Neck carcinoma & $0(n=9)$ \\
\hline Healthy volanteers & $12(n=50)$ \\
\hline
\end{tabular}

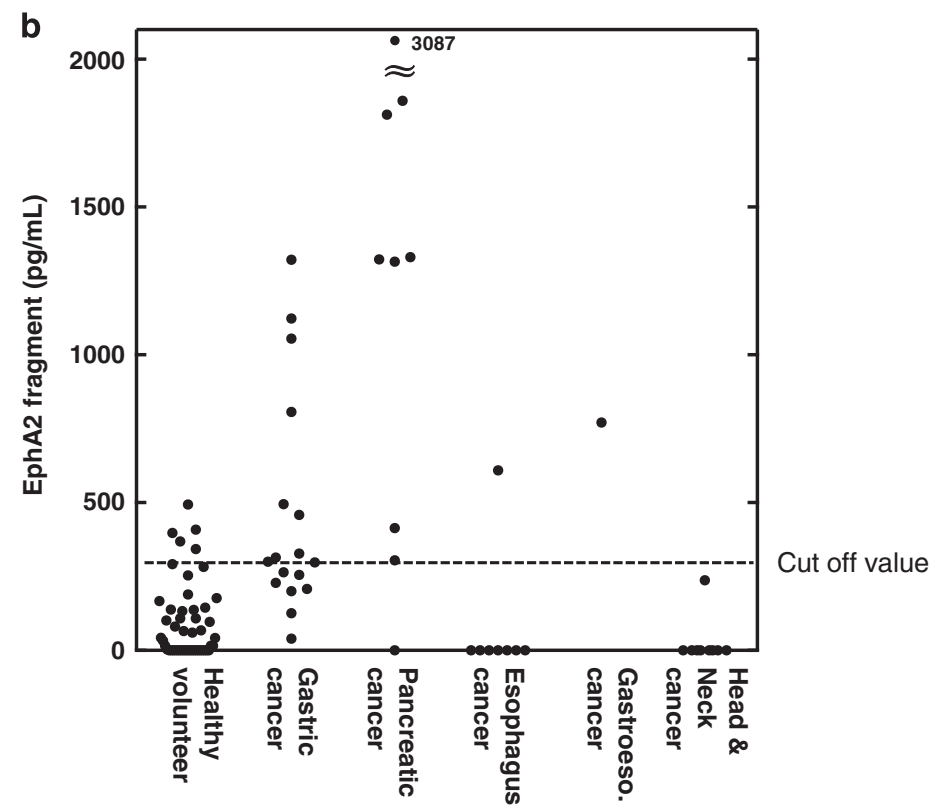

Figure 3 Sandwich ELISA. A sandwich ELISA using a combination of the mAb 76A1 as a capture antibody, and mAb 62A1 for detection was developed and used for this assay. (a) Standard curve for detecting an increasing amount of the soluble EphA2 fragment using antigen \#1 (0-250 pg/ml). (b) Concentrations of soluble EphA2 fragments in serum specimens from carcinoma patients and healthy donors. The cutoff value of $350 \mathrm{pg} / \mathrm{ml}$ was determined as the mean plus two S.D.s. (c) Positive ratios of serum specimens obtained from patients with different carcinomas and healthy donors

sera. The standard curve exhibited concentration-dependent linearity (Figure 3a). Reactivity did not saturate at least up to $10 \mathrm{ng} / \mathrm{ml}$ (data not shown). We then applied this method to analyze 94 serum samples from 17 gastric, nine pancreatic, eight esophageal, one gastroesophageal, and nine headand-neck carcinoma patients, and 50 healthy donors ( 26 men and 24 women; Figures $3 b$ and $c)$. The average soluble EphA2 fragment concentration obtained from healthy donors was $96 \pm 130 \mathrm{pg} / \mathrm{ml}$. Higher concentrations of the soluble EphA2 fragment were detected in patients with gastric carcinoma $(460 \pm 379 \mathrm{pg} / \mathrm{ml})$ and pancreatic carcinoma $(951 \pm 317 \mathrm{pg} / \mathrm{ml})$. On the basis of average values of the healthy donors, the cutoff value was set at $350 \mathrm{pg} / \mathrm{ml}$ (mean plus two S.D.s) to predict cancer from patient sera. It is of note that eight out of nine pancreatic cancer patients exhibited soluble EphA2 fragment levels over the cutoff value (Figures $3 b$ and $c$ and Supplementary Table).

Preliminary receiver operating characteristic curve analysis demonstrated that the area under the curve ratios for detecting the soluble EphA2 fragment in pancreatic and gastric cancer patients were 0.89 and 0.88 , respectively. The sensitivity and specificity for pancreatic cancer versus healthy donors were $89.0 \%$ and $90.0 \%$, respectively; $P<0.0002$ (Figure $4 \mathrm{a}$ ), and for gastric cancer were $88.2 \%$ and $84.0 \%$, respectively; $P<0.0001$ (Figure $4 \mathrm{~b}$ ). For comparison, we also measured values of CA19-9 as a conventional biomarker for digestive tumors, including pancreatic cancer (Figure 4c). The sensitivity and specificity of CA19-9 detection in sera from pancreatic cancer patients were $88.9 \%$ and $72.0 \%$, respectively; $P<0.0002$. The area under the curve ratio of CA19-9 detection in sera from pancreatic cancer patients was 0.83 . These findings suggest that the diagnostic accuracy of measuring levels of the soluble EphA2 fragment specific to pancreatic cancer exceeds that of CA19-9 (Figures $4 \mathrm{a}$ and $\mathrm{c}$ ).

\section{Discussion}

In normal tissues, EphA2 is expressed mainly in epithelial cells where MT1-MMP is not expressed. However, MT1-MMP is 
a ROC curve (Pancreatic ca vs HD)

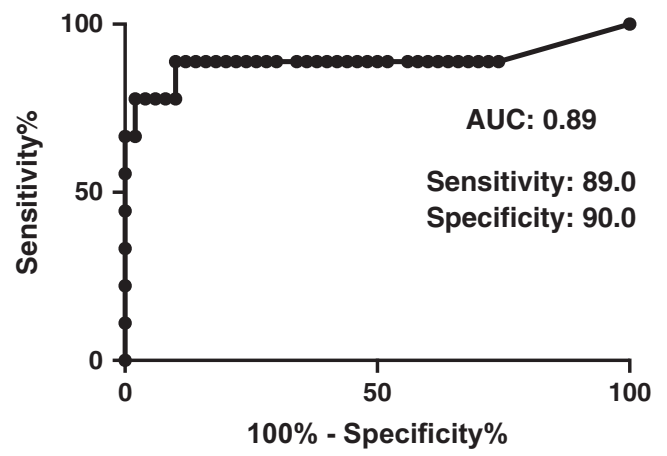

b ROC curve (Gastric ca vs HD)

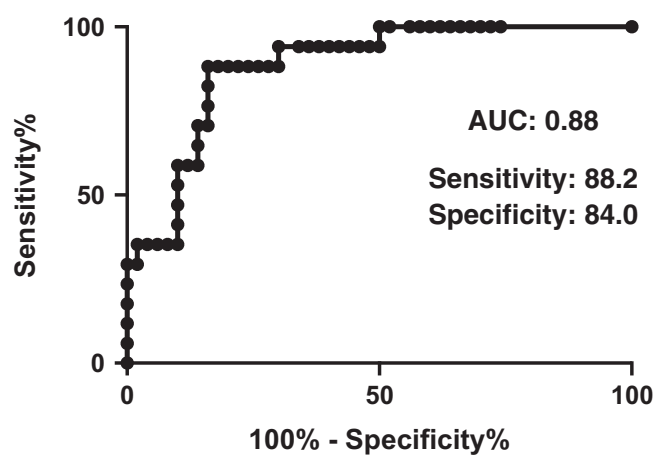

C ROC curve (Panreatic ca vs HD)

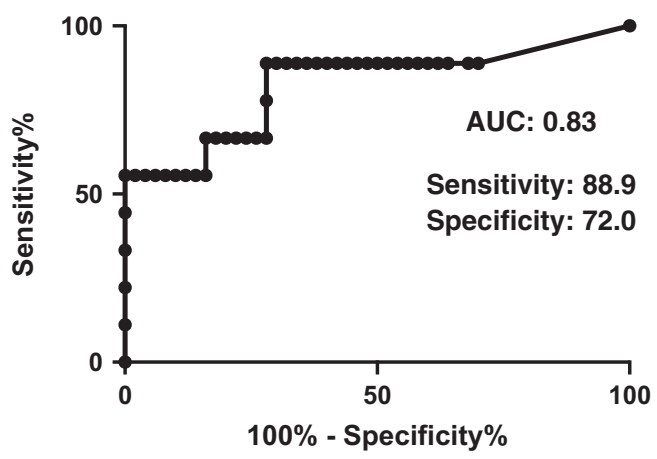

Figure 4 ROC curves. ROC curves associated with soluble EphA2 fragments in terms of detecting (a) pancreatic cancer (nine patients), (b) gastric cancer (17 patients), and healthy donors (HDs; 26 men and 24 women). (c) ROC curve associated with CA19-9 in terms of detecting pancreatic cancer (nine patients) and HDs

usually expressed in activated stromal cells, such as myofibroblasts, invading endothelial cells, and monocytes. ${ }^{19-21}$ Furthermore, MT1-MMP is frequently overexpressed in cancer cells together with EphA2 as reported in many previous studies and in the Cancer Genome Atlas database (http://cancergenome. nih.gov/). Expression of both proteins has been implicated in malignant behavior including aggressive growth, invasion, and metastasis of cancer cells. ${ }^{3,6,14}$ Notably, EphA2 is a substrate of MT1-MMP and can be processed on the surface of cancer cells. ${ }^{9,18}$ Proteolytic processing of EphA2 by MT1-MMP eliminates the EphA2 ligand-binding domain and converts it from a ligand-dependent tumor suppressor to a ligandindependent oncoprotein. ${ }^{9}$ Therefore, the proteolytic processing of EphA2 by MT1-MMP represents a cancer cell-specific event. This implies that the products of EphA2 cleavage could constitute markers for cancer progression.

To establish a method for detecting the products of EphA2 processing in cancer patients, we developed mAbs specific to the extracellular domain of EphA2 (antigen \#1). One of the three $\mathrm{mAbs}$ we developed, mAb 76A1, reacted specifically with the soluble EphA2 fragment, with negligible crossreactivity to the intact form (Figure 2). A quantitative sandwich ELISA was developed by taking the advantage of the specificity of the 76A1 mAb. We then applied this ELISA to commercially available serum specimens obtained from cancer patients and healthy donors. Higher levels of the soluble EphA2 fragment were observed in cancer patientderived sera compared with healthy donors. In particular, the levels for pancreatic and gastric cancers were significantly higher than the cutoff values, although this was not the case for esophageal and head-and-neck carcinoma samples. This suggests that, in addition to the expression of MT1-MMP and EphA2, other factors could be involved in releasing the EphA2 fragment into blood vessels from cancer cells.

Even though this analysis was preliminary in terms of the number of patients and the potential to improve the sensitivity of the ELISA, it was clearly evident that the soluble EphA2 fragment exists in blood from cancer patients and could be useful as a diagnostic biomarker for detecting pancreatic cancer. Although CA19-9 is frequently used for a clinical blood test to screen for pancreatic cancer, ${ }^{22,23}$ the soluble EphA2 fragment appears to be more accurate and has the potential to replace CA19-9 or be used in a complementary manner to overcome the limitations of CA19-9.

EphA2 includes both intact and cleaved forms secreted from cancer cells. ${ }^{9,18,24}$ In our preliminary results, high levels of EphA2 were detected frequently in serum from healthy donors $(382 \pm 493 \mathrm{pg} / \mathrm{ml}, n=26)$ when we used $\mathrm{mAb} 46 \mathrm{~A} 1$ that reacts with both soluble EphA2 fragments and the intact form (Supplementary Figure S2), whereas EphA2 values detected by $\mathrm{mAb} 76 \mathrm{~A} 1$ were almost negligible (Supplementary Figure S2). These results suggest that both EphA2 forms may exist in normal sera from healthy donors and, presumably, sera from cancer patients. It is likely that various EphA2 forms from non-cancerous cells diminish the sensitivity and specificity of that assay as a diagnostic tool for cancer patients.

On the basis of the results of our present study, we propose that the soluble EphA2 fragments shed into blood are a valuable biomarker for the presence of cancer cells in patients, particularly in pancreatic carcinoma. The development of mAbs that specifically recognize the soluble forms of EphA2 with negligible cross-reactivity to the intact forms is the key for accurate diagnosis. Although the number of patients subjected to this assay in our study was not large, the present results are sufficiently positive for testing a larger number of patient specimens using this assay method. We are improving the detection system further as a diagnostic tool and planning its evaluation in a larger patient cohort. Overall, specific detection of the soluble EphA2 fragment cleaved by MT1MMP in cancer cells is likely to become a key technology of pancreatic carcinoma diagnosis using blood specimens. 
Materials and Methods

EphA2 antibodies. The anti-EphA2 mAb 96-1 and polyclonal antibody (pAb) C309 were gifts from Daiichi Sankyo (Tokyo, Japan). The epitopes of mAbs 96-1 and pAb C309 were determined to be a ligand-binding and cysteine-rich domain, respectively. pAbs 371805 and C20 against EphA2 were purchased from R\&D Systems (Minneapolis, MN, USA) and Santa Cruz Biotechnology (Dallas, TX, USA), respectively. Epitopes of the pAbs 371805 and C20 consisted of EphA2 ligandbinding and cytoplasmic domains, respectively. All antibodies were applicable for immunoprecipitation assays, except pAb 309.

Recombinant antigens. Human EphA2 cDNA was purchased from Open Biosystems (Huntsville, AL, USA). Gene fragments corresponding to nucleotides 1-981 and 1-1578 of the coding region (antigens \#1 and 5) were amplified using the polymerase chain reaction, fused to a FLAG-tag-coding sequence at the $3^{\prime}$ end, and subcloned into the pCDNA3.2-DEST mammalian-expression vector using the Gateway System (Invitrogen, Carlsbad, CA, USA; Figure 1). Expression vectors for the EphA2 ligand-binding and cysteine-rich domains (antigen \#1) and EphA2 extracellular domain (antigen \#5) were transiently transfected under serum-free conditions into FreeStyle 293-F and A431 cells, respectively. These recombinant antigens were secreted into serum-free conditioned medium, collected, and purified by anti-FLAG mAb-conjugated agarose-affinity chromatography as described previously. ${ }^{25}$ Purity of the recombinant proteins was verified by SDS-PAGE with Coomassie Brilliant Blue staining.

For antibody epitope mapping, antigens \#2, 3, and 4, corresponding to EphA2 aa 28-125, 101-250, and 226-328, respectively, with C-terminal FLAG tags, were amplified using the polymerase chain reaction and subcloned into the $\mathrm{pET} 160$ vector using the Gateway System (Invitrogen; Figure 1). Recombinant antigens were expressed in $E$. coli and purified with anti-FLAG mAb-conjugated agarose chromatography as described previously. ${ }^{21}$

Immunization, hybridization, and hybridoma screening. BALB/C mice were injected intraperitoneally with $50 \mu \mathrm{g}$ of recombinant soluble EphA2 fragments containing the ligand-binding domain (antigen \#1) and emulsified in an equal volume of complete Freund's adjuvant (Difco, Sparks, MD, USA). Immunization was boosted twice at 2-week intervals with equal amounts of antigen \#1 with incomplete Freund's adjuvant (Difco). Six weeks after the third immunization, mice were injected intravenously with the same amount of antigen \#1 without adjuvant. Three days after this injection, blood was collected and centrifuged at $1500 \times g$ at room temperature to obtain serum for use as a positive control (that is, containing antibodies specific to antigen \#1) during hybridoma screening.

Hybridization of the P3U1 mouse myeloma cell line and spleen cells was performed according to methods described by IBL (Gunma, Japan). Hybridoma cells were cultured in HAT selection medium for 10 days, and the cells were re-seeded into 96-well plates. Supernatants from each well containing growing cells were examined for antibody production using antigen \#1 and an ELISA. Limited dilution and cloning of cells was repeated twice. Three hybridoma clones producing mAbs reactive to the soluble EphA2 fragment (mAbs 46A1, 62A1, and 76A1) were obtained. To analyze the epitopes of these mAbs, western blotting using recombinant antigens \#2, 3 , and 4 was performed under non-reducing and reducing conditions.

Immunoprecipitation. The soluble EphA2 fragment (antigen \#1; $1 \mu \mathrm{g}$ ), uncleaved extracellular domain of EphA2 (antigen \#5; $1 \mu \mathrm{g}$ ), or intact EphA2 from the A431 cell lysate were incubated overnight at $4{ }^{\circ} \mathrm{C}$ with mAbs or pAbs in radioimmunoprecipitation assay buffer (final volume $200 \mu \mathrm{l}$ ). Forty microliters of suspended Protein-G magnetic beads were added and incubated at $4{ }^{\circ} \mathrm{C}$ for $1 \mathrm{~h}$ with agitation. The beads were washed three times with radioimmunoprecipitation assay buffer and those containing antigen/antibody complexes were removed with a magnetic rack. Precipitated antigen-antibody complexes were released from the beads by addition of $2 x$ sodium dodecyl sulfate sample buffer containing $5 \%(v / v)$ $\beta 2$-mercaptoethanol. Details are described in our previous report. ${ }^{25}$

Sandwich ELISA. The 96-well plates were coated with mAb $76 \mathrm{~A} 1$ or $46 \mathrm{~A} 1$ $(20 \mu \mathrm{g} / \mathrm{ml})$ in phosphate-buffered saline overnight at $4{ }^{\circ} \mathrm{C}$. Pre-immune mouse serum was used as the negative control. The wells were blocked with protein-free blocking buffer (Pierce Biotechnology, Rockford, IL, USA) for $1 \mathrm{~h}$ and dried for $40 \mathrm{~min}$ at room temperature. Each well was washed three times with phosphate-buffered saline containing $0.05 \%$ Tween-20, followed by adding $0-1000 \mathrm{pg} / \mathrm{ml}$ of the MT1-MMP. cleaved fragment (antigen \#1) to the wells for reaction with biotin-conjugated mAb
$62 \mathrm{~A} 1$ for $2 \mathrm{~h}$ at $37^{\circ} \mathrm{C}$. After washing, bound antigens were detected with horseradish peroxidase-labeled Avidin-D $(0.1 \mu \mathrm{g} / \mathrm{ml})$. The absorbance/reference $(590 / 620 \mathrm{~nm})$ of the wells filled with $3,3^{\prime}, 5,5^{\prime}$-tetramethylbenzidine, after stopping the reaction with $1 \mathrm{~N}$ $\mathrm{H}_{2} \mathrm{SO}_{4}$, was measured using an iMark microplate reader (Bio-Rad, Hercules, CA, USA). Detailed methods were described previously. ${ }^{26}$

Clinical specimens. Pancreatic carcinoma sera (nine cases) were obtained from ProMedDx (Norton, MA, USA). Gastric (17 cases), esophageal (8 cases), gastroesophageal ( 1 case), and head-and-neck (9 cases) cancer sera, and sera from 50 healthy donors (26 men and 24 women) were purchased from Kokusai Bio (Tokyo, Japan). Information about serum specimens is shown in the Supplementary Table. All serum specimens were used after centrifugation at $1500 \times \mathrm{g}$ for $15 \mathrm{~min}$ at $4{ }^{\circ} \mathrm{C}$. Levels of the conventional digestive tumor marker, CA19-9, were measured using a chemiluminescence immunoassay in serum specimens from cancer patients and healthy donors. ${ }^{23}$

Statistical analysis. Data were analyzed using GraphPad Prism 6 software (GraphPad Software, La Jolla, CA, USA). A $P$-value of $<0.05$ was considered statistically significant by the Mann-Whitney U-test.

\section{Conflict of Interest}

The authors declare no conflict of interest.

Acknowledgements. We thank Jun Hasegawa (Daiichi Sankyo, Tokyo Japan) for helpful discussions. N Koshikawa was supported by a Grant-in-Aid for Scientific Research on Innovative Areas (17H06327) from the Ministry of Education, Culture, Sports, Science, and Technology of Japan (MEXT), by a Grant-in-Aid for Scientific Research (C) (17K09027), and a Core-to Core program (Establishing International Research Network of Mathematical Oncology) from the Japan Society for the Promotion Science (JSPS; 963179). M Seiki was supported by a Grant-in-Aid for Scientific Research (S) from the MEXT (22220014). N N Koshikawa received research funding from Daiichi Sankyo (Tokyo, Japan) and Abbott Laboratories (North Chicago, IL, USA).

\section{Publisher's Note}

Springer Nature remains neutral with regard to jurisdictional claims in published maps and institutional affiliations.

1. Ogawa K, Pasqualini R, Lindberg RA, Kain R, Freeman AL, Pasquale EB. The ephrin-A1 ligand and its receptor, EphA2, are expressed during tumor neovascularization. Oncogene 2000; 19: 6043-6052.

2. Cheng N, Brantley DM, Liu H, Lin Q, Enriquez M, Gale N et al. Blockade of EphA receptor tyrosine kinase activation inhibits vascular endothelial cell growth factor-induced angiogenesis. Mol Cancer Res 2002; 1: 2-11.

3. Biao-xue R, Xi-guang C, Shuan-ying Y, Wei L, Zong-juan M. EphA2-dependent molecular targeting therapy for malignant tumors. Curr Cancer Drug Targets 2011; 11: 1082-1097.

4. Ireton $\mathrm{RC}$, Chen J. EphA2 receptor tyrosine kinase as a promising target for cancer therapeutics. Curr Cancer Drug Targets 2005; 5: 149-157.

5. Miao H, Li DQ, Mukherjee A, Guo H, Petty A, Cutter $\mathrm{J}$ et al. EphA2 mediates liganddependent inhibition and ligand-independent promotion of cell migration and invasion via a reciprocal regulatory loop with Akt. Cancer Cell 2009; 16: 9-20.

6. Beauchamp A, Debinski W. Ephs and ephrins in cancer: ephrin-A1 signalling. Semin Cell Dev Biol 2012; 23: 109-115.

7. Cui XD, Lee MJ, Yu GR, Kim IH, Yu HC, Song EY et al. EFNA1 ligand and its receptor EphA2: potential biomarkers for hepatocellular carcinoma. Int J Cancer 2010; 126: 940-949.

8. Beauchamp A, Lively MO, Mintz A, Gibo D, Wykosky J, Debinski W. EphrinA1 is released in three forms from cancer cells by matrix metalloproteases. Mol Cell Biol 2012; 32: 3253-3264.

9. Koshikawa N, Hoshino D, Taniguchi H, Minegishi T, Tomari T, Nam SO et al. Proteolysis of EphA2 converts it from a tumor suppressor to an oncoprotein. Cancer Res 2015; 75: 3327-3339.

10. Tomari T, Koshikawa N, Uematsu T, Shinkawa T, Hoshino D, Egawa N et al. High throughput analysis of proteins associating with a proinvasive MT1-MMP in human malignant melanoma A375 cells. Cancer Sci 2009; 100: 1284-1290.

11. Tatsukawa R, Koga K, Aoki M, Koshikawa N, Imafuku S, Nakayama J et al. Immunohistochemical demonstration of EphA2 processing by MT1-MMP in invasive cutaneous squamous cell carcinoma. Virchows Arch 2016; 469: 25-34.

12. Itoh Y. Membrane-type matrix metalloproteinases: their functions and regulations. Matrix Biol 2015; 44-46: 207-223.

13. Sakamoto T, Seiki M. Integrated functions of membrane-type 1 matrix metalloproteinase in regulating cancer malignancy: beyond a proteinase. Cancer Sci 2017; 108: 1095-1100. 
14. Seiki M, Koshikawa N, Yana I. Role of pericellular proteolysis by membrane-type 1 matrix metalloproteinase in cancer invasion and angiogenesis. Cancer Metastasis Rev 2003; 22 : 129-143.

15. Macrae M, Neve RM, Rodriguez-Viciana P, Haqq C, Yeh J, Chen C et al. A conditional feedback loop regulates Ras activity through EphA2. Cancer Cell 2005; 8: 111-118.

16. Gingras D, Bousquet-Gagnon N, Langlois S, Lachambre MP, Annabi B, Beliveau R. Activation of the extracellular signal-regulated protein kinase (ERK) cascade by membranetype-1 matrix metalloproteinase (MT1-MMP). FEBS Lett 2001; 507: 231-236.

17. Liang K, Liu F, Sun J, Liu C, Lyon C, Bernard D et al. Nanoplasmonic quantification of tumour-derived extracellular vesicles in plasma microsamples for diagnosis and treatment monitoring. Nat Biomed Eng 2017; 1: 0021.

18. Sugiyama N, Gucciardo E, Tatti O, Varjosalo M, Hyytiainen M, Gstaiger M et al. EphA2 cleavage by MT1-MMP triggers single cancer cell invasion via homotypic cell repulsion. $J$ Cell Biol 2013; 201: 467-484.

19. Schram K, Wong MM, Palanivel R, No EK, Dixon IM, Sweeney G. Increased expression and cell surface localization of MT1-MMP plays a role in stimulation of MMP-2 activity by leptin in neonatal rat cardiac myofibroblasts. J Mol Cell Cardiol 2008; 44: 874-881.

20. Yana I, Sagara H, Takaki S, Takatsu K, Nakamura K, Nakao K et al. Crosstalk between neovessels and mural cells directs the site-specific expression of MT1-MMP to endothelial tip cells. J Cell Sci 2007; 120: 1607-1614.

21. Klose A, Zigrino P, Mauch C. Monocyte/macrophage MMP-14 modulates cell infiltration and T-cell attraction in contact dermatitis but not in murine wound healing. Am J Pathol 2013; 182: 755-764.

22. De Rosa A, Cameron IC, Gomez D. Indications for staging laparoscopy in pancreatic cancer. HPB 2016; 18: 13-20.

23. O'Brien DP, Sandanayake NS, Jenkinson C, Gentry-Maharaj A, Apostolidou S, Fourkala EO et al. Serum CA19-9 is significantly upregulated up to 2 years before diagnosis with pancreatic cancer: implications for early disease detection. Clin Cancer Res 2015; 21: 622-631.
24. Tauro BJ, Greening DW, Mathias RA, Ji H, Mathivanan S, Scott AM et al. Comparison of ultracentrifugation, density gradient separation, and immunoaffinity capture methods for isolating human colon cancer cell line LIM1863-derived exosomes. Methods 2012; 56: 293-304.

25. Koshikawa N, Minegishi T, Sharabi A, Quaranta V, Seiki M. Membrane-type matrix metalloproteinase-1 (MT1-MMP) is a processing enzyme for human laminin gamma 2 chain. J Biol Chem 2005; 280: 88-93.

26. Kamada M, Koshikawa N, Minegishi T, Kawada C, Karashima T, Shuin T et al. Urinary laminin-gamma2 is a novel biomarker of non-muscle invasive urothelial carcinoma. Cancer Sci 2015; 106: 1730-1737.

(i) Cell Death and Disease is an open-access journal published by Nature Publishing Group. This work is licensed under a Creative Commons Attribution 4.0 International License. The images or other third party material in this article are included in the article's Creative Commons license, unless indicated otherwise in the credit line; if the material is not included under the Creative Commons license, users will need to obtain permission from the license holder to reproduce the material. To view a copy of this license, visit http://creativecommons.org/licenses/by/4.0/

(C) The Author(s) 2017

Supplementary Information accompanies this paper on Cell Death and Disease website (http://www.nature.com/cddis) 